\title{
Mathematical Modelling of Deforestation of Forested Area Due to Lack of Awareness of Human Population and Its Conservation
}

\author{
Ararso Hussen Teru ${ }^{1}$, Purnachandra Rao Koya ${ }^{2}$ \\ ${ }^{1}$ Department of Mathematics, Wolkite University, Wolkite, Ethiopia \\ ${ }^{2}$ Department of Mathematics, Wollega University, Nekemte, Ethiopia \\ Email address: \\ ararso.hussen@wku.edu.et (A. H. Teru),drkpraophd@gmail.com (P. R. Koya)

\section{To cite this article:} \\ Ararso Hussen Teru, Purnachandra Rao Koya. Mathematical Modelling of Deforestation of Forested Area Due to Lack of Awareness of \\ Human Population and Its Conservation. Mathematical Modelling and Applications. Vol. 5, No. 2, 2020, pp. 94-104. \\ doi: 10.11648/j.mma.20200502.15
}

Received: January 3, 2020; Accepted: March 10, 2020; Published: May 12, 2020

\begin{abstract}
As the density of human population increases the forest density will be highly affected by population from time to time for agricultural, industrial, economic purpose, and etc. Because of lack of awareness about the importance of forestry resources, the human populations clear forests for different purposes. Keeping this in mind, a nonlinear mathematical model is proposed and analyzed to study the deforestation of forest resources due to lack of clear information about utility of the forest as well as to increase forestry resources by plantation on the conservation of forestry resources. The model is in the form of ordinary differential equations. The result of this study shows that as the density of population as well as population pressure increases, the cumulative density of forest resources decreases. Reversely, the test of supporting human awareness on the importance of forest resources for global purposes show that as awareness of human population increases the declaration of forest resources decreases. In addition to this, increasing the density of forest resources through plantation may replace the clear-cut of forest area. This help the conservation carried out to in force the pressure of human population to save the forest density and forest habit. For these findings analytical and numerical analyses are performed.
\end{abstract}

Keywords: Mathematical Model, Logistic Growth Model, Forest Density, Population Density, Population Pressure, Numerical Simulation

\section{Introduction}

Forests are the dominant terrestrial ecosystem of Earth, and are distributed across the globe (Wikipedia). Also it is defined as land spanning more than 0.5 hectares with trees higher than 5 meters and a canopy cover of more than 10 percent, or trees able to reach these thresholds. It does not include land that is predominantly under agricultural or urban land use [29].

As is well known, forests assist in the global cycling of water, oxygen, carbon and nitrogen, as well as forming the habitat for many wildlife species. It gives many benefits for human, as fuel source, water storage, recreation area, industrial raw material, etc.

The WRI, 1997 estimates that only one-fifth of the world's original forest cover remains. The annual rate of forest loss has increased significantly from 2.7 million ha between 1990 and 2000 to 6.3 million ha between 2000 and 2005 . According to FAO 2010 report, forest area is decreasing 4 million ha per year due to human activities. Therefore, to enhance the forest resources along with fulfilling the growing demand of their products is a challenging problem faced by all societies [13].

It is widely observed that population pressure contributes to the deterioration and depletion of important natural resources in developing countries. Growing rural populations and rising subsistence demands have resulted in the expansion of cultivated onto increasingly marginal soils and have shortened periods of fallow, leading to erosion and the loss of soil fertility [21].

To maintain forestry resources, the government of Ethiopia has introduced 740 million plant plantation programs day on July, 2019. This day around 300 million plants were planted 
by citizens of Ethiopia.

In recent years, numerous models have been developed to assess the depletion of forestry resources. The key role of each model is to estimate the level of forest biomass due to population density as well as population demand in forestry environment. They have considered a model for interactions between forestry biomass (resources density), wildlife population, human population, population pressure, industrialization pressure as well as alternative conservation. A nonlinear mathematical model of forestry biomass depletion due to human population, population pressure and industrialization was studied. Analysis of the model shows that the equilibrium level of forest resources decreases as the equilibrium level in density of human population, population pressure and industrialization increase. It is found that if the crowding by industrialization increases, then biomass density of forestry resources decreases. Therefore, it is necessary to control industrialization to protect the forestry resources stability [28]. In the depletion of forestry resources caused by population and population pressure augmented industrialization. By this study they conclude that the equilibrium density of resource biomass decreases as the equilibrium densities of population and industrialization increase. The mathematical modeling of effects of population and population pressure on forest resources and their conservation was studied. It is assumed that the cumulative density of forest resources and the density of populations follow logistic models with prey-predator type nonlinear interaction terms. It is considered that the carrying capacity of forest resources decreases by population pressure. A conservation model is also proposed to control the population pressure by providing some economic incentives to people, the amount of which is assumed to be proportional to the population pressure [3]. The model analysis shows that as the density of population or population pressure increases, the cumulative density of forest resources decreases, and the resources may become extinct if the population pressure becomes too large. It is also noted that by controlling the population pressure, using some economic incentives, the density of forest resources can be maintained at an equilibrium level, which is population density dependent. A thesis titled with a mathematical model to achieve sustainable forest management was also studied to describe the effect of forest resources due to population and population pressure. In the modeling process, it is assumed that due to increase in population size, the demand of population or population pressure for forest products, lands, etc., increases and to reduce this population pressure, economic efforts are employed proportional to the population pressure. They assumed that technological efforts in the form of genetically engineered plants are applied proportional to the depleted level of forest resources to conserve them. Model analysis reveals that increase in economic and technological efforts increases the density of forest resources but further increase in these efforts destabilizes the system. Numerical simulation is carried out to verify analytically findings and explore the effect of different parameters on the dynamics of model system [1]. Keeping in mind the above discussions, we propose and analysis the depletion of forest resources due to population pressure and its reforestation. According to this thesis the main focus is to decrease the population pressure by giving awareness for the population about effectiveness of the forest and reducing the problem by new plantation. It is more similar with modeling the effect of economic efforts to control population pressure and conserve forestry resources [30]. But they are differing on model formulation and number of variable of studies. In case of previous study the economic incentive is taken as conservation variable, where as in our case plantation is considered as rate of plantation to increase the density of forest resources. Moreover, this thesis is similar with a mathematical model to achieve sustainable forest management research paper. But in model formulation there are some modification of parameters and number of conservation variables. In a mathematical model to achieve sustainable forest management the researchers use both economic incentive and technological effort as conservation variable [1]. Therefore the main difference of this thesis from the two studies is the number of variable of studies. In our case we reduce both economic incentive and technological effort variable and consider plantation as rate of plantation on the dimension of forest density that added on forest dynamic equation.

In this study, the main assumption was that as population increases, the demand of population or population pressure increases. To fulfill this demand of population, the forestry resources are overexploited by population to clear forest land and agricultural land increases, this ultimately leads to deforestation [11]. Hence, the situation of deforestation mainly occurs due to the human related activities. Human population uses the forestry resources and forest land for various purposes.

Therefore, to cope up the problem of deforestation, our aim in the present study is to assess the impact of reforestation by plantation on forestry resources areas. The second aim is to identify the effect of awareness of population about advantage and disadvantage of deforestation of forest area.

\section{Model Formulation}

In this study the density of forest resource is basically considered as the variable of study which is affected by population density and population pressure. Therefore, the variables of studies included in the model are

i. $B(t)$ denotes the cumulative density of forest resources

ii. $N(t)$ denotes the human population density

iii. $P(t)$ denotes the Population pressure

To model the dynamics of forest density dependent on population density and population pressure, the following assumptions have been made:

i. The growth rates of forestry resource and population density are assumed to be following logistic growth model.

ii. The growth of forest resources is in the direct proportion of population density and population pressure.

Forest Density Equation: Since human population uses the forest resources for their daily needs of food, fuel, medicinal 
plants, fodder, etc., it is assumed that forest resources are depleted due to increase in human population $[1,12]$.

With increase in human population, the demand of population increases. Due to this population pressure, population move in the forest region and clear forest for construction of housing complexes, agricultural purposes, space for cattle population, etc. Thus, increase in population pressure $(\mathrm{P})$ affects the carrying capacity of forest resources. In view of this, we have assumed that forest resources are depleted due to increase in the population pressure [1]. In the modeling process, the dynamics of the forest biomass density is assumed to be governed by logistic equation as follows:

$$
\frac{d B}{d t}=\alpha B\left(1-\frac{B}{L}\right)-\beta B N-B^{2} P+\rho B, B(0)=B_{0} \geq 0
$$

Here in (1), $\alpha$ denotes intrinsic growth rate of forest biomass, $\mathrm{L}$ is carrying capacity of forest biomass, $\beta$ represents the depletion rate coefficient of forest biomass due to human population density, $\gamma$ the depletion rate coefficients of forest resources due to population pressure and $\rho$ indicates new plantation to cover forestresource area by forest.

Population Density Equation: It is assumed that the growth of human population due to forest biomass is directly proportional to the densities of forest biomass as well as human population i.e. $\beta B N[24]$. Also there are growth rate coefficients of forest human population in forest depleted area by population pressure $\left(\mu \gamma B^{2} N\right)$. In view of these assumptions; the differential equation governing the dynamics of the population is given by;

$$
\frac{d N}{d t}=\delta N\left(1-\frac{N}{K}\right)+\beta B N+\mu \gamma B^{2} N, N(0)=N_{0} \geq 0
$$

Here in (2), $K$ and $\delta$ is the carrying capacity and the intrinsic growth rate of human population respectively. The constants $\beta$ the growth rate of cumulative density of populations due to forest resources.

Population Pressure Equation: The demand of human population for forest resources increases as human population increases, so it is assumed that increase in population pressure is directly proportional to the density of human population $[12,24]$. Now, with the passage of time few people become aware about the dreadful effects of the depletion of forest resources, so they avoid the use of forest resources. Thus, it is assumed that population pressure depletes naturally [1]. These assumptions indicates that the population pressure equation is given by

$$
\frac{d P}{d t}=\theta N-\sigma P, P(0)=P_{0} \geq 0
$$

Here in (3), $\theta$ is the growth rate coefficient of population pressure due to population density and $\sigma$ is the depletion rate coefficient of population pressure due to awareness of the population on importance of forestry resources.

Thus, the dynamics of the system of model equation is governed by the following set of nonlinear ordinary differential equations

$$
\frac{d B}{d t}=\alpha B\left(1-\frac{B}{L}\right)-\beta N B-\gamma B^{2} P+\rho
$$

$$
\begin{gathered}
\frac{d N}{d t}=\delta N\left(1-\frac{N}{K}\right)+\beta B N+\mu \gamma B^{2} \\
\frac{d P}{d t}=\theta N-\sigma
\end{gathered}
$$

Also, the initial conditions are given by

$$
B(0)=B_{0} \geq 0, N(0)=N_{0} \geq 0, P(0)=P_{0} \geq 0
$$

\section{Model Analysis}

In this section the boundedness of the solutions is proved and hence shown that the model developed here is mathematically valid and biologically meaningful. Furthermore, equilibrium points have been identified and their stability analysis is conducted.

\subsection{Boundedness of Solutions}

Region or domain of attraction is a set gives a measure of how far from the equilibrium point the trajectory can start and still converge to it. In general, the region of attraction cannot be computed analytically. However, what we can do is estimate it, using for instance, Lyapunov functions.

Lemma: The set

$$
\Omega=\left\{(B, N, P): 0 \leq B \leq L_{m}, 0 \leq N \leq N_{m}, 0 \leq P \leq \frac{\theta}{\sigma} N_{m}\right\}
$$

is a region of attraction for all solutions initiating in the interior of the positive octant of the model system (4). Here $L_{m}=\frac{L}{\alpha}(\alpha+\rho)$, and $N_{m}=\frac{K}{\delta}\left(\delta+\beta L+\mu \gamma L^{2}\right)[1,3$, 12, 24, 28].

Proof: Consider first equation of model system (4)

$$
\frac{d B}{d t}=\alpha B\left(1-\frac{B}{L}\right)-\beta B N-\gamma B^{2} P+\rho B
$$

$0 \leq \alpha B\left(1-\frac{B}{L}\right)-\beta B N-\gamma B^{2} P+\rho B \leq \alpha B\left(1-\frac{B}{L}\right)+\rho B$

Hence,

$$
0 \leq \alpha B\left(1-\frac{B}{L}\right)+\rho B
$$

Simplifying

$$
B \leq \frac{L}{\alpha}(\alpha+\rho)
$$

By definition,

$$
\begin{gathered}
0 \leq B_{0} \leq B \\
\Rightarrow 0 \leq B(t) \leq \frac{L}{\alpha}(\alpha+\rho)=L_{m}
\end{gathered}
$$

From second equation of (4),

$$
\frac{d N}{d t}=\delta N\left(1-\frac{N}{K}\right)+\beta B N+\mu \gamma B^{2} N
$$

Since change of population density with change of time $0 \leq \frac{d N}{d t}$ and $B \leq L$, we have; 


$$
0 \leq \delta N\left(1-\frac{N}{K}\right)+\beta B N+\mu \gamma B^{2} N
$$

This implies that,

$$
0 \leq N(t) \leq \frac{K}{\delta}\left(\delta+\beta L_{m}+\mu \gamma L_{m}^{2}\right)=N_{m}
$$

From assumption of population density

$$
\left(0 \leq \frac{d P}{d t}\right) .
$$

Also from model system (4) and the maximum value of population density $N_{m}$, we get;

$$
0 \leq \frac{d P}{d t}=\theta N-\sigma P \leq \lambda N_{m}-\lambda_{0} P
$$

Hence,

$$
0 \leq P(t) \leq \frac{\theta}{\sigma} N_{m}
$$

The $\Omega$ shows all the solutions of model are non-negative and bounded, and hence the model is biologically wellbehaved [11].

\subsection{Equilibrium Analysis}

The equilibrium points are derived by;

$$
\begin{aligned}
& \frac{d B}{d t}=\alpha B\left(1-\frac{B}{L}\right)-\beta B N-\gamma B^{2} P+\rho B=0 \quad \text { the form } \\
& \frac{d N}{d t}=\delta N\left(1-\frac{N}{K}\right)+\beta B N+\mu \gamma B^{2} N=0 \\
& f(B)=\alpha\left(1-\frac{B}{L}\right)-\beta N-\gamma B P+\rho=\alpha\left(1-\frac{B}{L}\right)-\beta \frac{K}{\delta}\left(\delta+\beta B+\mu \gamma B^{2}\right)-\gamma B \frac{K \theta}{\delta \sigma}\left(\delta+\beta B+\mu \gamma B^{2}\right)+\rho
\end{aligned}
$$

We now that the boundary of $B(t)$ is

$$
0 \leq B(t) \leq \frac{L}{\alpha}(\alpha+\rho)
$$

Substituting the boundary values in (14), we have;

$$
f(0)=\alpha\left(1-\frac{0}{L}\right)-\beta \frac{K}{\delta}\left(\delta+\beta .0+\mu \gamma .0^{2}\right)-\gamma \cdot 0 \cdot \frac{K \theta}{\delta \sigma}\left(\delta+\beta .0+\mu \gamma .0^{2}\right)+\rho
$$

Thus, $f(0)=\alpha-\beta K+\rho>0$ provided that, $\alpha+\rho>\beta K$

$$
\begin{aligned}
& f\left(L_{m}\right)=\alpha\left(1-\frac{L_{m}}{L}\right)-\beta \frac{K}{\delta}\left(\delta+\beta \cdot L_{m}+\mu \gamma \cdot L_{m}{ }^{2}\right)-\gamma \cdot L_{m} \cdot \frac{K \theta}{\delta \sigma}\left(\delta+\beta \cdot L_{m}+\mu \gamma \cdot L_{m}{ }^{2}\right)+\rho<0 \\
& f^{\prime}(B)=-\left[\frac{\alpha}{L}+\frac{K \beta}{\delta}(\beta+2 \mu \gamma B)+\frac{K \theta \gamma}{\delta \sigma}\left(\delta+\beta B+\mu \gamma B^{2}\right)+\frac{K \theta \gamma}{\delta \sigma} B(\beta+2 \mu \gamma B)\right]<0
\end{aligned}
$$

From (15), (16) and (17), we conclude that there is a when $\alpha+\rho>\beta K$. unique $\hat{B}$ such that $f(\widehat{B})=0$.

Then substituting $\hat{B}$ in equation;

$$
\delta\left(1-\frac{N}{K}\right)+\beta B+\mu \gamma B^{2}=0
$$

We get $\widehat{N}$, and substituting it in; $\theta N-\sigma P=0$ Evaluating it with respect to $P$; we have arrive on $\widehat{P}$. Thus, the interior equilibrium point $E_{4}(\widehat{B}, \widehat{N}, \widehat{P})$ exists,

\subsection{Stability Analysis}

The local stability behavior of $E_{1}, E_{2}, E_{3}$ and $E_{4}$ are investigated by determining the sign of Eigen-values of the corresponding Jacobean matrix. From the model system (4), the Jacobean matrix is given by 


$$
J(B, N, P)=\left[\begin{array}{ccc}
\alpha-\frac{2 \alpha}{L} B-\beta N-2 \mu B P+\rho & -\beta B & -2 \gamma B^{2} \\
\beta N+2 \mu \gamma B N & \delta-\frac{2 \delta}{K} N+\beta B+\mu \gamma B^{2} & 0 \\
0 & \theta & -\sigma
\end{array}\right]
$$

i. Stability analysis of $E_{1}(0,0,0)$

The Jacobean matrix of $E_{1}$ is;

$$
J(0,0,0)=\left[\begin{array}{ccc}
\alpha+\rho & 0 & 0 \\
0 & \delta & 0 \\
0 & \theta & -\sigma
\end{array}\right]
$$

Thus, the determinant of $\lambda I-J$ is given by;

$$
\begin{gathered}
\qquad \begin{array}{ccc}
\lambda-(\alpha+\rho) & 0 & 0 \\
0 & \lambda-\delta & 0 \\
0 & \theta & \lambda+\sigma
\end{array} \mid=0 \\
J\left(\frac{L}{\alpha}(\alpha+\rho), 0,0\right)=\left[\begin{array}{cc}
-\alpha-\rho & - \\
0 & \delta+L \\
0
\end{array}\right. \\
\text { Thus, the determinant of } \lambda I-J \text { is given by; } \\
\left|\begin{array}{ccc}
\lambda+(\alpha+\rho) & \frac{L \beta}{\alpha}(\alpha+\rho) & \frac{2 L^{2} \gamma}{\alpha^{2}}(\alpha+\rho)^{2} \\
0 & \lambda-\left(\delta+\frac{L \beta}{\alpha}(\alpha+\rho)\right) & 0 \\
0 & \lambda & \lambda+\sigma
\end{array}\right|=0
\end{gathered}
$$$$
J\left(\frac{L}{\alpha}(\alpha+\rho), 0,0\right)=\left[\begin{array}{ccc}
-\alpha-\rho & -\frac{L \beta}{\alpha}(\alpha+\rho) & -\frac{2 L^{2} \gamma}{\alpha^{2}}(\alpha+\rho)^{2} \\
0 & \delta+\frac{L \beta}{\alpha}(\alpha+\rho) & 0 \\
0 & \theta & -\sigma
\end{array}\right]
$$

We have, $\lambda_{1}=-(\alpha+\rho), \lambda_{2}=\delta+\frac{L \beta}{\alpha}(\alpha+\rho)$ and $\lambda_{3}=$ $-\sigma$.

Since $\lambda_{2}=\delta+\frac{L \beta}{\alpha}(\alpha+\rho)>$, equilibrium point $E_{2}$ is unstable.

Stability analysis of $E_{3}\left(0, K, \frac{\theta}{\sigma} K\right)$ :

The Jacobean matrix at $E_{3}$ is;

$$
J\left(0, K, \frac{\theta}{\sigma} K\right)=\left[\begin{array}{ccc}
\alpha-K \beta+\rho & 0 & 0 \\
K \beta & -\delta & 0 \\
0 & \theta & -\sigma
\end{array}\right]
$$

Thus, the determinant of $\lambda I-J$ is given by;

$$
\left|\begin{array}{ccc}
\lambda-(\alpha-K \beta+\rho) & 0 & 0 \\
K \beta & \lambda+\delta & 0 \\
0 & \theta & \lambda+\sigma
\end{array}\right|=0
$$

We have, $\lambda_{1}=\alpha-K \beta+\rho, \lambda_{2}=-\delta$ and $\lambda_{3}=-\sigma$

Since $\lambda_{1}=\alpha-K \beta+\rho>0$, equilibrium point $E_{3}$ is unstable.

iii. Stability analysis of $E_{4}$

The stability behavior of interior equilibrium $E_{3}$ is not obvious from the corresponding Jacobean matrix. To determine the sign of Eigen-values of the Jacobean matrix $J$ is difficult, so we use the Routh-Hurtiz criterion or Lyapunov's theory to determine the stability behavior of interior equilibrium $E_{4}$. The following theorems give
The characteristic equation is;

$$
(\lambda-(\alpha+\rho))(\lambda-\delta)(\lambda+\sigma)=0
$$

This indicate that the Eigen-values at $E_{1}$ are $\lambda_{1}=\alpha+\rho$, $\lambda_{2}=\delta$ and $\lambda_{3}=-\sigma$.

Since $\lambda_{1}$ and $\lambda_{2}$ are positive Eigen-values, then $E_{1}$ unstable.

ii. Stability analysis of $E_{2}\left(\frac{L}{\alpha}(\alpha+\rho), 0,0\right)$ :

The Jacobean matrix is given by;

sufficient conditions for local and global stability behavior of equilibrium $E_{4}$.

Theorem 1: The interior equilibrium $E_{4}$ if exists (conditional), is locally asymptotically stable provided the following inequalities hold [1, 3, 12, 24, 28, 30, 31]:

$$
\frac{\gamma^{2} \widehat{B}^{2}}{\sigma^{2}\left(\frac{\alpha}{L}+\gamma \widehat{P}\right)}<\frac{16}{9} \frac{\beta \delta}{K \theta^{2}(\beta+\mu \gamma \widehat{B})}
$$

Proof: Now, to determine the nature of Eigen-values of Jacobin matrix $B$ (evaluated at $E_{4}$ ) isdifficult by using Routh-Herwitz criterion, so we use Lyapunov's stability theory. By using the Taylor's series expansion, the linearized system of model system (4) about $E_{4}(\widehat{B}, \widehat{N}, \widehat{P})$ can be written as follows:

$$
\left[\begin{array}{c}
\dot{x} \\
\dot{y} \\
\dot{z}
\end{array}\right]=\left[\begin{array}{ccc}
-\left(\frac{\alpha}{L}+\gamma \hat{P}\right) \hat{B} & -\beta \widehat{B} & -\gamma \hat{B}^{2} \\
\beta \widehat{N}+\mu \gamma \hat{B} \widehat{N} & -\frac{\delta}{K} \widehat{N} & 0 \\
0 & \theta & -\sigma
\end{array}\right]\left[\begin{array}{l}
x \\
y \\
z
\end{array}\right]
$$

Where "." denote $\frac{d}{d t}$ and $x, y, z$ are small perturbations around the equilibrium $E_{4}$. i.e.

$$
B=\widehat{B}+x, N=\widehat{N}+y \text { and } P=\widehat{P}+z .
$$

Now consider the positive definite function which is given as follows:

$$
X=\frac{1}{2}\left(\frac{x^{2}}{\widehat{B}}+k_{1} \frac{y^{2}}{\widehat{N}}+k_{2} z^{2}\right)
$$

Differentiating (25) with respect to time $t$ and substituting (23) and (24), where $k_{1}$ and $k_{2}$ are some positive constants to be chosen appropriately.

$$
\frac{d X}{d t}=-\left(\frac{\alpha}{L}+\gamma \hat{P}\right) x^{2}-k_{1} \frac{\delta}{K} y^{2}-k_{2} \sigma z^{2}-\left(\beta-k_{1}(\beta+\mu \gamma \hat{B}) x y-\gamma \hat{B} x z+k_{2} \theta y z\right.
$$


Choosing $k_{1}=\frac{\beta}{\beta+\mu \gamma \hat{B}}$ and $\frac{d X}{d t}$ simplified as;

$$
\frac{d X}{d t}=-\left(\frac{\alpha}{L}+\gamma \hat{P}\right) x^{2}-\frac{\beta \delta}{K(\beta+\mu \gamma \widehat{B})} y^{2}-k_{2} \sigma z^{2}-\gamma \widehat{B} x z+k_{2} \theta y z
$$

Now, if the following inequalities hold:

$$
\begin{aligned}
(\gamma \hat{B})^{2} & <\frac{4}{3} k_{2} \sigma\left(\frac{\alpha}{L}+\gamma \hat{P}\right) \\
k_{2} \theta^{2} & <\frac{4}{3} \frac{\beta \delta \sigma}{K(\beta+\mu \gamma \hat{B})}
\end{aligned}
$$

Then $\frac{d X}{d t}$ will be negative definite. From inequalities (28)(29) we may easily choose the positive value of $k_{2}$;

$$
\begin{aligned}
& \frac{3}{4} \frac{\gamma^{2} \hat{B}^{2}}{\sigma\left(\frac{\alpha}{L}+\gamma \hat{P}\right)}<k_{2}<\frac{4}{3} \frac{\beta \delta \sigma}{K \theta^{2}(\beta+\mu \gamma \hat{B})} \\
& \text { Thus, } \frac{\gamma^{2} \hat{B}^{2}}{\sigma^{2}\left(\frac{\alpha}{L}+\gamma \hat{P}\right)}<\frac{16}{9} \frac{\beta \delta}{K \theta^{2}(\beta+\mu \gamma \hat{B})} \\
& W=\left(B-\widehat{B}-\hat{B} \ln \frac{\hat{B}}{B}\right)+k_{1}\left(N-\widehat{N}-\widehat{N} \ln \frac{\widehat{N}}{N}\right)+k_{2} \frac{(P-\hat{P})^{2}}{2}
\end{aligned}
$$

Where $m_{1}, m_{2}>0$ and will be chosen appropriately. It can be easily checked that the function $W$ is zero at the equilibrium $E_{4}(\widehat{B}, \widehat{N}, \widehat{P})$ and positive for all otherpositive values of $B, N$ and $P$.

Now the derivative of $W$ with respect to $t$ along the trajectories of model system (4) is given by

$$
\begin{gathered}
\frac{d W}{d t}=(B-\widehat{B}) \frac{d B}{d t}+m_{1}(N-\widehat{N}) \frac{d N}{d t}+m_{2}(P-\widehat{P}) \frac{d P}{d t} \\
\frac{d W}{d t}=-\left(\frac{\alpha}{L}+\gamma \hat{P}\right)(B-\widehat{B})^{2}-m_{1} \frac{\delta}{K}(N-\widehat{N})^{2}-m_{2} \sigma(P-\widehat{P})^{2} \\
-\left(\beta-m_{1}(\beta+\mu \gamma \widehat{B})(B-\widehat{B})(N-\widehat{N})-\gamma \widehat{B}(B-\widehat{B})(P-\widehat{P})+m_{2} \theta(N-\widehat{N})(P-\widehat{P})\right.
\end{gathered}
$$

Choosing $m_{1}=\frac{\beta}{\beta+\mu \gamma \hat{B}}$ and $\frac{d X}{d t}$ simplified as;

$$
\frac{d W}{d t}=-\left(\frac{\alpha}{L}+\gamma \hat{P}\right)(B-\hat{B})^{2}-m_{1} \frac{\delta}{K}(N-\widehat{N})^{2}-m_{2} \sigma(P-\hat{P})^{2}-\gamma \widehat{B}(B-\widehat{B})(P-\hat{P})+m_{2} \theta(N-\widehat{N})(P-\hat{P})
$$

Now $\frac{d W}{d t}$ will be negative definite inside the region of attraction $\Omega$, provided the following inequalities:

$$
\begin{aligned}
\frac{\gamma^{2} L^{2}(\alpha+\rho)^{2}}{\alpha^{2}} & <\frac{4}{3} m_{2} \sigma\left(\frac{\alpha}{L}+\gamma \hat{P}\right) \\
m_{2} \theta^{2} & <\frac{4}{3} \frac{\beta \delta \sigma}{K(\beta+\mu \gamma \hat{B})}
\end{aligned}
$$

Solving for $m_{2}$ from (37)-(38), we have

$$
\frac{\gamma^{2} L^{2}(\alpha+\rho)^{2}}{\alpha^{2} \sigma^{2}\left(\frac{\alpha}{L}+\gamma \hat{P}\right)}<\frac{16}{9} \frac{\beta \delta}{K \theta^{2}(\beta+\mu \gamma \widehat{B})}
$$

Remark: For smaller values of $\gamma, \mu$, and for larger values of $\sigma$ the stability of theorems 1) and 2) above are easily fulfilled. If these stability conditions of interior equilibrium fail to satisfy, then the coexistence of the systems destabilized.

\section{Numerical Analysis}

In this section, we simulate model system (4) by choosing the following set of parameter values, which are given as follows:

Thus, $\frac{d W}{d t}$ is negative definite under condition (39).

Table 1. The approximate values of parameters [1, 12].

\begin{tabular}{llllll}
\hline No. & Parameters & Estimation values & No. & Parameters & Estimated values \\
\hline 1. & $L$ & 80 & 6. & $K$ & 100 \\
2. & $\alpha$ & 1 & 7. & $\delta$ & 0.6 \\
3. & $\beta$ & 0.003 & 8. & $\mu$ & 0.04 \\
4. & $\gamma$ & 0.007 & 9. & $\theta$ & 0.015 \\
5. & $\rho$ & 0.012 & 10. & $\sigma$ & 0.4 \\
\hline
\end{tabular}

From non-zero isoclines we have the cubic function; 


$$
\begin{gathered}
f(B)=\alpha\left(1-\frac{B}{L}\right)-\beta \frac{K}{\delta}\left(\delta+\beta B+\mu \gamma B^{2}\right)-\gamma B \frac{K \theta}{\delta \sigma}\left(\delta+\beta B+\mu \gamma B^{2}\right)+\rho \\
=(\alpha-\beta K+\rho)-\left(\frac{\alpha}{L}+\frac{\beta^{2} K}{\delta}+\frac{\gamma \theta K}{\sigma}\right) B-\left(\frac{\beta \mu \gamma K}{\delta}+\frac{\beta \gamma \theta K}{\delta \sigma}\right) B^{2}-\frac{\mu \gamma^{2} \theta K}{\delta \sigma} B^{3}
\end{gathered}
$$

Substituting the values of parameters in (41) and solving for $B$, we have three roots $-18.7525+58.5820 i$, $-18.7525-58.5820 i$ and 15.3621 .

Since $0 \leq B(t) \leq \frac{L}{\alpha}(\alpha+\rho)=101.5$, then

$$
\widehat{B}=15.3621
$$

Substituting in $\widehat{N}=\frac{K}{\delta}\left(\delta+\beta \widehat{B}+\mu \gamma \widehat{B}^{2}\right)=118.6941087658$ and substituting

$$
\widehat{P}=\frac{\theta}{\sigma} \widehat{N}=4.4510290787
$$

Thus, the interior equilibrium point of the model is given by:

$$
(\widehat{B}, \widehat{N}, \hat{P})=(15.3621,118.6941087658,4.4510290787)
$$

$$
J(\widehat{B}, \widehat{N}, \widehat{P})=\left[\begin{array}{ccc}
\alpha-\frac{2 \alpha}{L} \hat{B}-\beta \widehat{N}-2 \gamma \hat{B} \hat{P}+\rho & -\beta \widehat{B} & -\gamma \hat{B}^{2} \\
\beta \widehat{N}+2 \mu \gamma \hat{B} \widehat{N} & \delta-\frac{2 \delta}{K} \widehat{N}+\beta \widehat{B}+\mu \gamma \widehat{B}^{2} & 0 \\
0 & \theta & -\sigma
\end{array}\right]=\left[\begin{array}{ccc}
-0.6854 & -0.0461 & -1.6520 \\
1.3772 & -0.7122 & 0 \\
0 & 0.015 & -0.4
\end{array}\right]
$$

Then the Eigen-values of the Jacobean matrix are $-0.8274,-0.4851+0.2695 i$ and $-0.4851-0.2695 i$.

Here it is noted that the Eigen-values of $J(\widehat{B}, \widehat{N}, \hat{P})$ are either negative or with negative real part. Therefore, the negativity of the sign ensures the local stability of interior equilibrium $E_{4}$ for the above set of parameter values.

From the data in the table the model boundary;

$$
0 \leq B(t) \leq L_{m}=80.96,0 \leq N(t) \leq N_{m}=446.3577 \text { and } 0 \leq P(t) \leq \frac{\theta}{\sigma} N_{m}=16.7384 .
$$

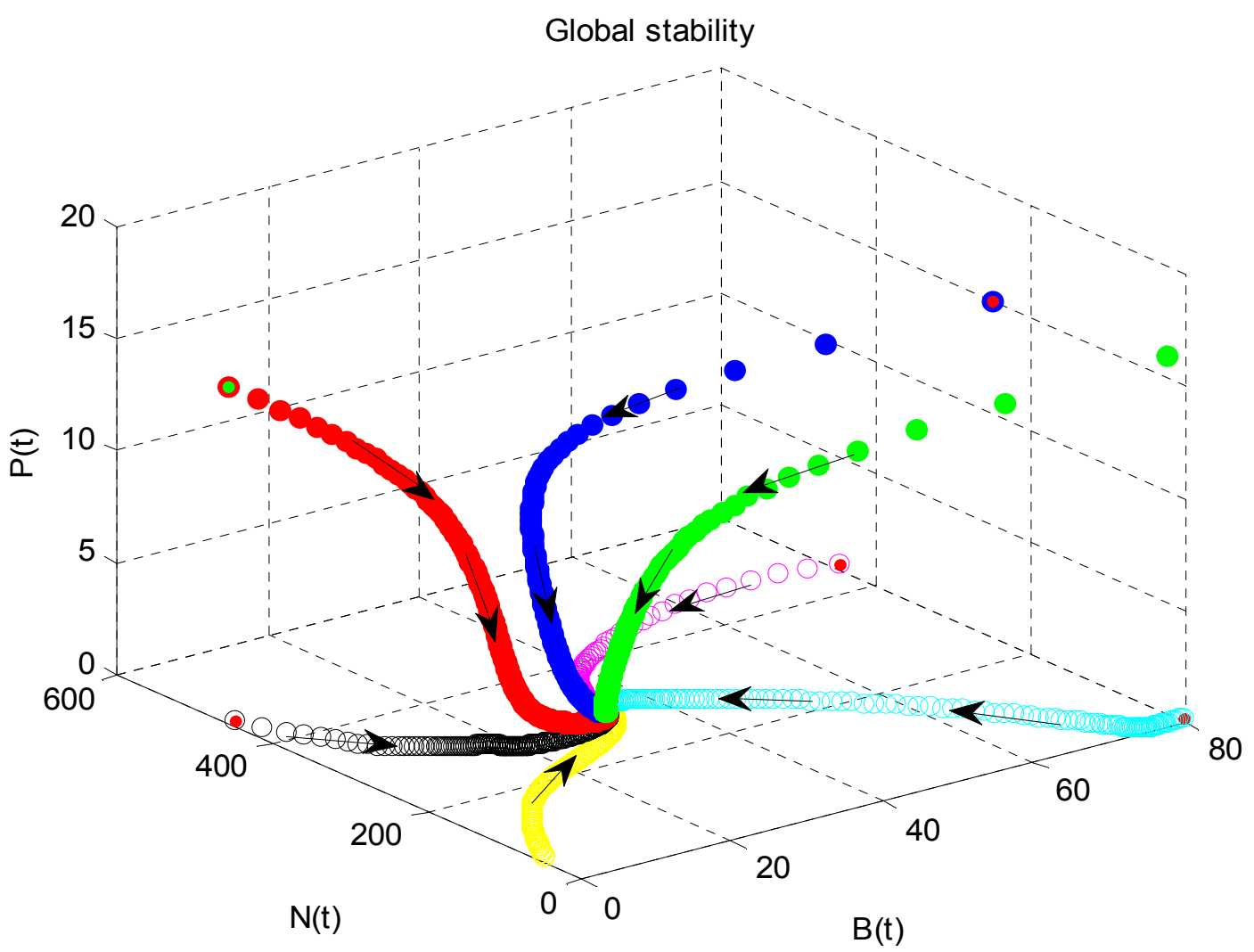

Figure 1. Global stability of $E_{4}$ in $B-N-P$ plane. 
These values of parameters satisfy the global stability conditions of Theorem 2. The trajectories of $B, N$ and $P$ with different initial values are plotted in Figure 1. From this figure, we note that all the trajectories starting from different initial conditions converge to the equilibrium point. This shows that is globally asymptotically stable.

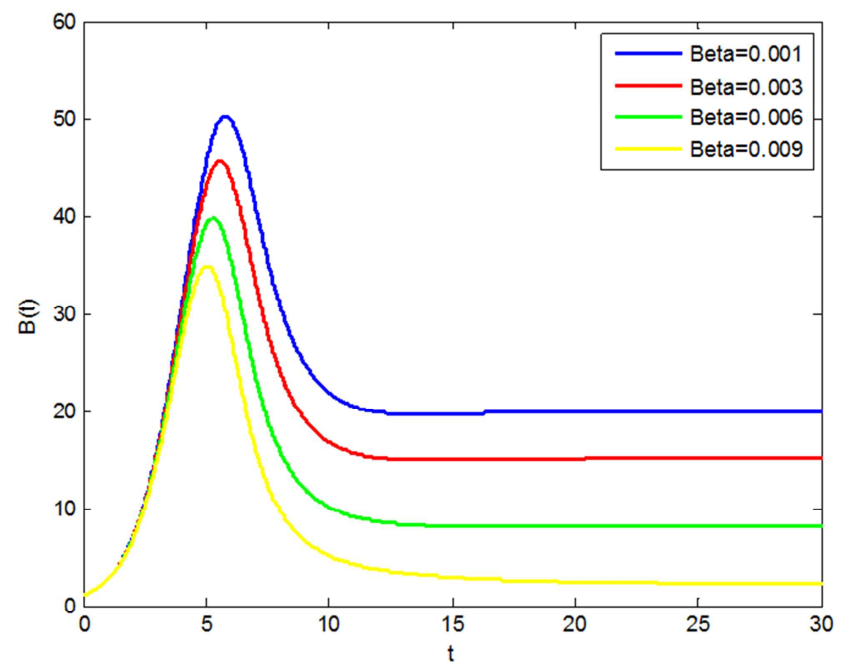

a)
It may be noted here that $\beta, \gamma, \rho, \mu$ and $\sigma$ are important parameters governing the objective of the dynamics of the system. Therefore, we have numerical simulated and interpret the behavior of $B$ with respect to time for different values of parameters.

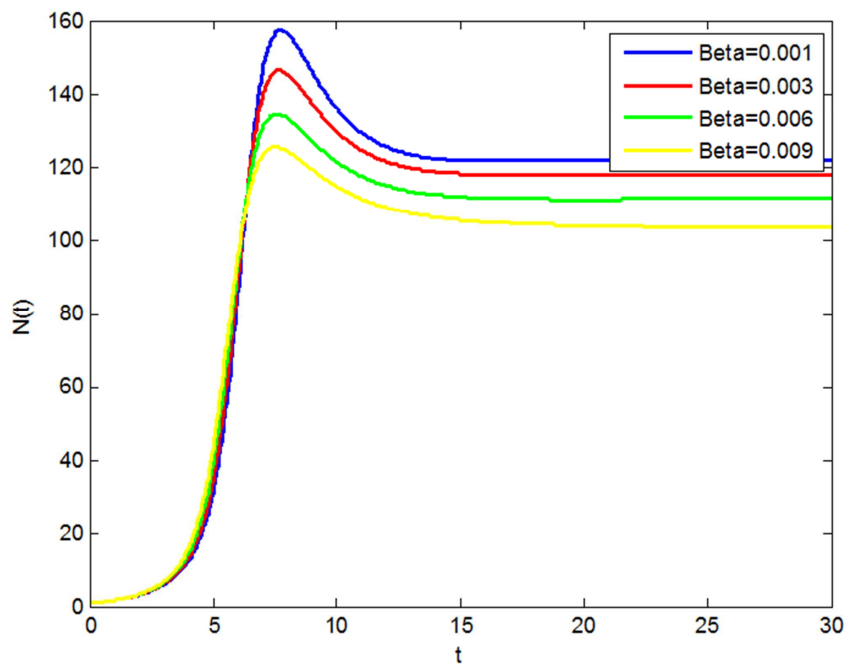

b)

Figure 2. Plot of variation of $B$ with respect to time for different values of $\beta$.

The forest resource biomass $B$ initially increases for $\beta$ less than its optimal level and then tends to its equilibrium points. It decrease for $\beta$ is less than its optimal level and it may tends to zero (figure 2). This shows that if the population

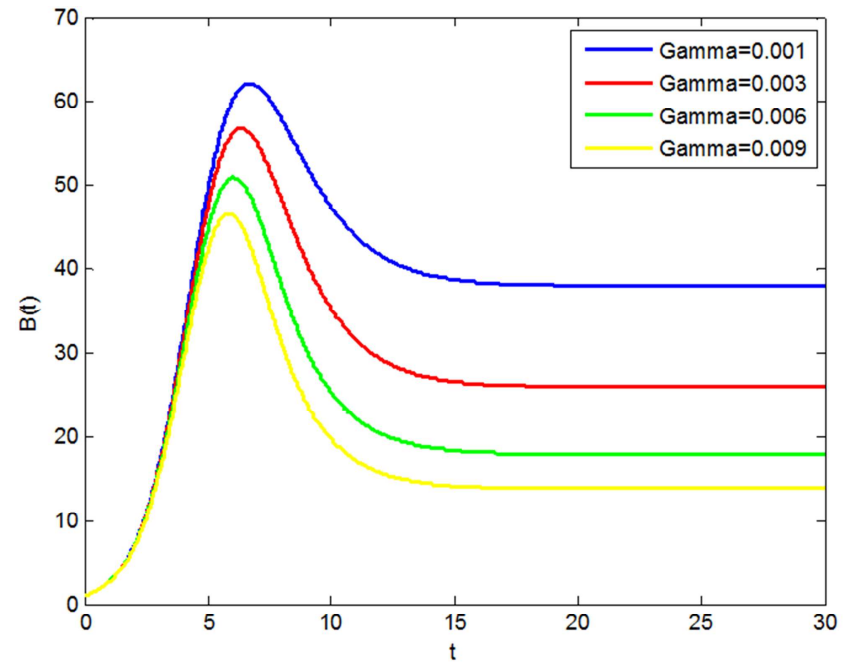

a) utilizes the resource without any control, then the resource biomass decreases and become changed into land without forest. In the absence of population density the forest density highly increases.

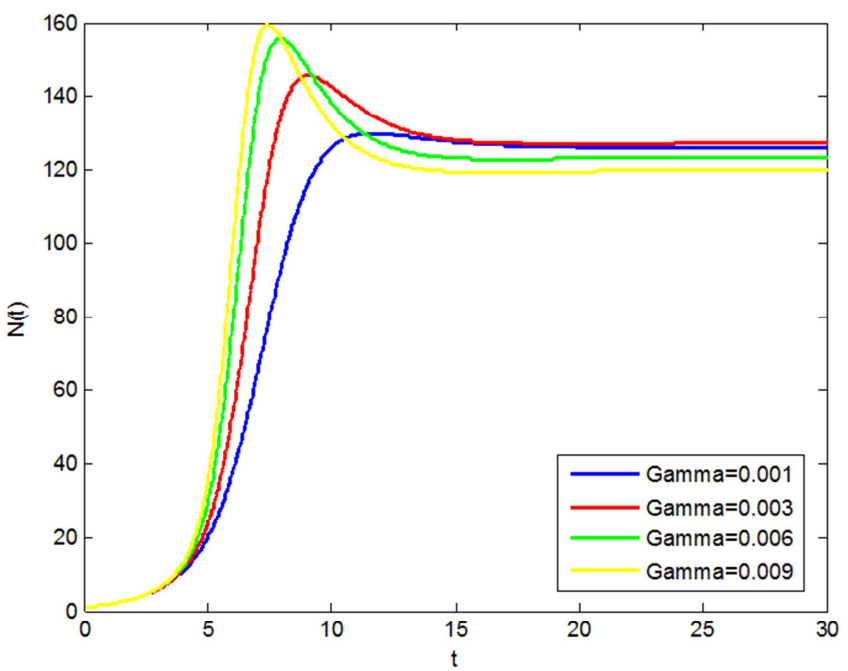

b)

Figure 3. Plot of variation of $B$ with respect to time for different values of $\gamma$.

The density of forest resources $B$ decreases continuously and becomes tends to zero level as $\gamma$ increases. But if the value of $\gamma$ decrease and lastly tends to zero the forest densityincreases with the constant rate. This means the carrying capacity of forest resources affected by population pressure if depletion rate $\gamma$ of carrying capacity of forest resources due to population pressure increases continuously. 


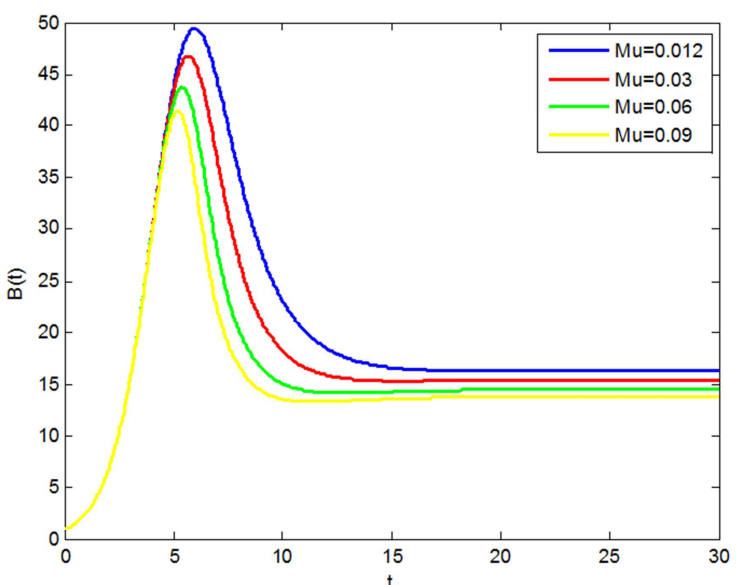

a)

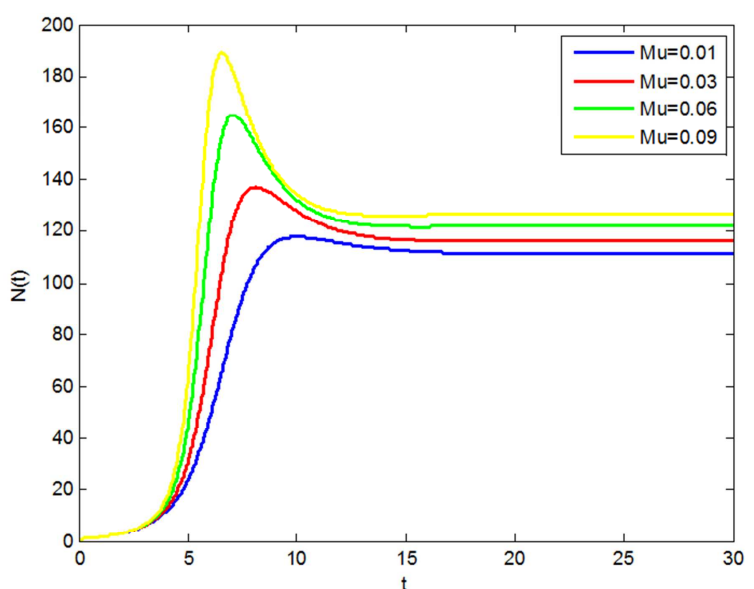

b)

Figure 4. Effect of different values of $\sigma$ on $B$ andNwith time $t$.

We observe from figure 4 that as the values of $\mu$ increases the human population density highly increases and lastly tends to its equilibrium level. This indicates as the carrying

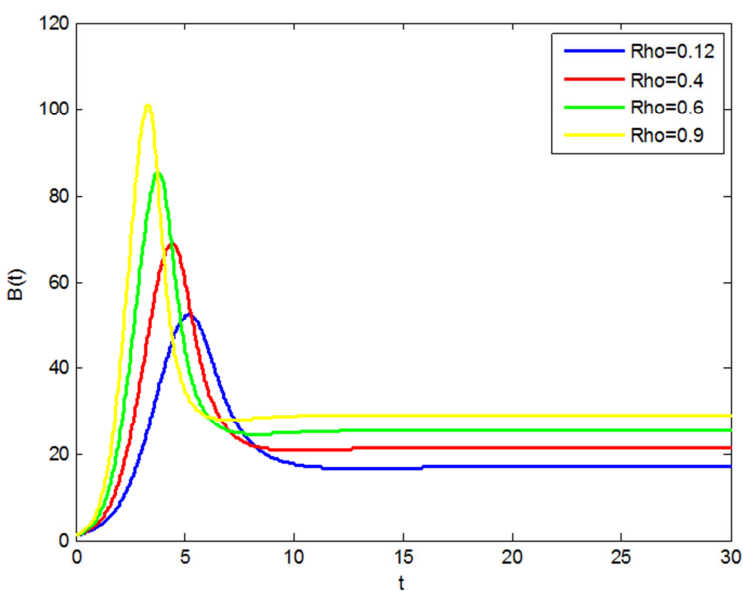

a) capacity of forest biomass affected by population pressure, the area may facilitate the condition to human density population to increases.

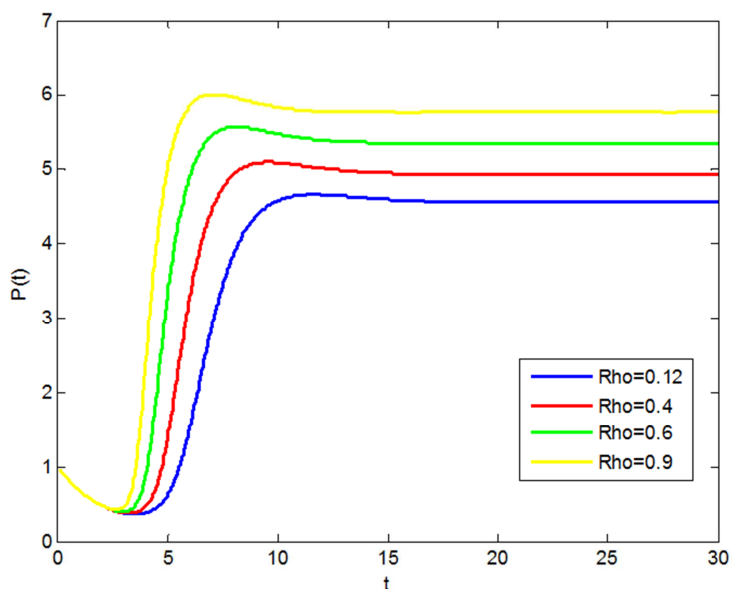

b)

Figure 5. Effect of different values of $\rho$ on the forestry resources and Population pressure with time.

From Figure5a) and b), it is manifested that the equilibrium levels of forestry resources and population pressure increases as $\rho$ increases. This indicates that without

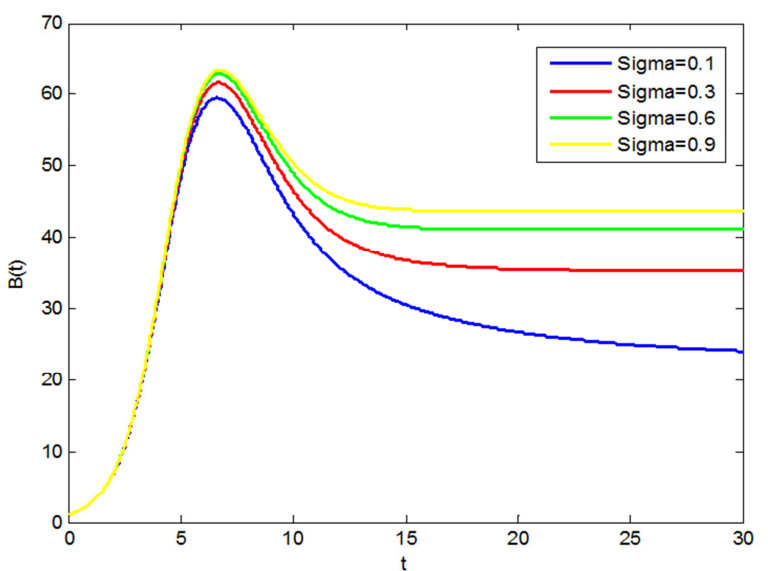

a) controlling population pressure, we may decrease the problem of clearance of the forest from forestry resources by increasing the density of the forest through plantation.

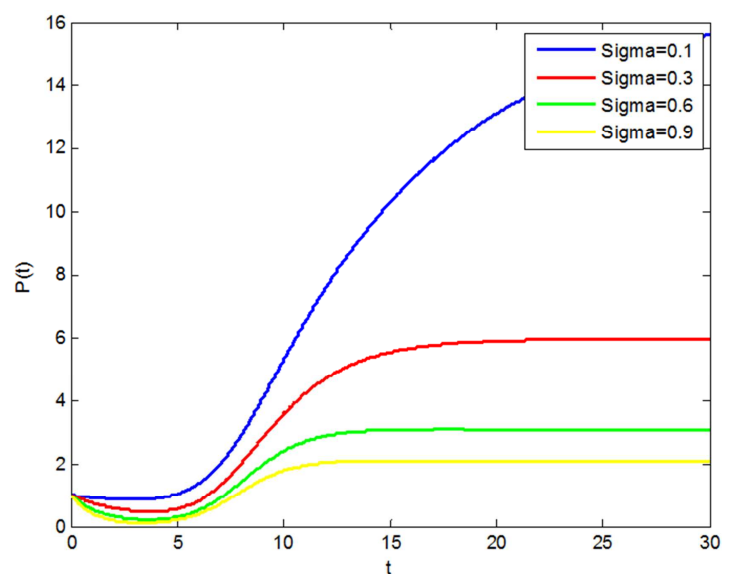

b)

Figure 6. Effect of different values of $\sigma$ on $B$ andP with time $t$ 
From Figure 6a) and b) as the value of $\sigma$ increases the equilibrium level of forestry resources density increases, and also the equilibrium of population pressure decrease respectively. Hence, we may conclude that as the awareness of the human population about the effectiveness of forest biomass increases the population pressure on forest resources decreases. And so forest density increases.

\section{Conclusion}

As is well known, forests assist in the global cycling of water, oxygen, carbon and nitrogen, as well as forming the habitat for many wildlife species. It gives many benefits for human, as fuel source, water storage, recreation area, industrial raw material, etc.

It is widely observed that population pressure contributes to the deterioration and depletion of important natural resources in developing countries. Growing rural populations and rising subsistence demands have resulted in the expansion of cultivated onto increasingly marginal soils and have shortened periods of fallow, leading to erosion and the loss of soil fertility [21].

So to study this problem, we have considered that forestry resources and human population follow logistic growth in their natural habitats, and we consider due to population density increase the population pressure increase is another study factor, which depletes the forestry resources. Depending on these variables of studies the mathematical model of three dimensional ordinary differential equations, which contains the correlation of those variables (whether directly or indirectly) was formulated.

We use the local and global stability of the equilibrium point of the system using Lyapunov's stability theorem. The result shows that if depletion of forest resources due to population and population pressure increases continuously without any conservation or control method, it results in decreasing of equilibrium level of forest resources. In other case, if there is high reforestation of forest area the density of forest recourses may survive for long period of time. Moreover, if the awareness of the population about importance of forest resources increases from time to time, the enforcement of population on forest area may decrease and the density of forest density may stay at its optimal level.

To discuss the applicability of the model, a numerical result of the data is also presented. It indicates that the level of forest biomass can be maintained at appropriate level if there is high re-plantation and giving awareness for population near forest area.

\section{References}

[1] A. K. Misra and Kusum Lata, A mathematical model to achieve sustainable forest management Vol. 6, No. 4 (2015) 1550040 (18 pages).

[2] A. K. Misra, Kusum Lata, Depletion and Conservation of
Forestry Resources: A Mathematical Model, January (2015) 23 (1): $25-41$.

[3] A. K. Misra, Kusum Lata and J. B. Shukla, Effects of population and population pressureon forest resources and their conservation: a modeling study, (2014) 16: 361-374.

[4] Misra, AK and Lata, Kusum and Shukla, JBA mathematical model for the depletion offorestry resources due to population and population pressure augmented industrialization, vol. $5, \mathrm{n}$. 01, p. 1350022, (2014).

[5] Shukla, JB and Lata, Kusum and Misra, AK (2011), modeling the depletion of a renewable resource by population and industrialization: Effect of technology on its conservation.

[6] Abdurahiman Kubsa, Granting exclusive user rights to the forest dwellers in the state owned forest: the WAJIB approach in Ethiopia.

[7] AbrahaHatsey, Tend of forest cover change in ethiopia; Paper presented in South AfricaGeorg-August University Gottingen, September, 2015.

[8] Math 4330, Numerical Solution of ODEs Using Matlab.

[9] Andam, Emmanuel Appoh, Analysis of transmission of dynamics Tuberculosis (TB) usingdifferential equations: A case study of Amansie west district, Ghana, 2013.

[10] ArarsaRegassa, DidhaDiriba, GedefaNegera, Bale Mountains Eco-region Reduction ofEmission from Deforestation and Forest Degradation (REDD+) Project Ethiopia.

[11] Atasi Patra (2013), Dynamics of Biological Species: Some Mathematical Models, December, 2013.

[12] B. Dubey, S. Sharma, P. Sinha and J. B. Shukla, Modelling the depletion of forestry resources by population and population pressure augmented industrialization, AppliedMathematical Modelling 33 2009, 3002-3014.

[13] FekaduGurmessa, Forest loss and climate change in Ethiopia, Vol. 4 (5), pp. 216-224, May. 2015.

[14] GirmaAmente. Integrated and participatory forest management in the Bale Mountainsof Ethiopia, 2006.

[15] LudekBerec. Mathematical modeling in ecology and epidemiology July 2011.

[16] Manju Agarwal, RachanaPathak, Conservation of Forestry Biomass with the Use of Alternative Resource, Open Journal of Ecology, 2015, 5, 87-109.

[17] Manju Agarwal, Tazeen Fatima and H. I. Freedman, Depletion of forestry resourcebiomass due to industrialization pressure: a ratio-dependent mathematical model, Journal of Biological Dynamics, 2010 4: 4 381-396.

[18] Mette LoycheWilkie, VialedelleTerme di Caracalla Global forest resources assessment 2010 country report Ethiopa $\mathrm{FAO}$ Forestry Department Rome 00153, Italy (2010).

[19] MulugetaLemenih and HabtemariamKassa, Re-Greening Ethiopia: History, Challenges and Lessons Forests 2014, 5, 1896-1909; doi: 10.3390/f5081896.

[20] Nadhurah BT Abdul Halim, Tuberculosis Model: A mathematical analysis, 2015. 
[21] Netsanet Deneke, Land use and Land Cover changes in Harenna Forest and Surrounding area, Bale Mountain national park, 2007.

[22] Robert Repet to and Thomas Holmes, The Role of Population in Resource Depletion inDeveloping Countries, Vol. 9, No. 4 (Dec., 1983), pp. 609-632.

[23] Shyam Sundar, Effect of Elevated Carbon Dioxide Concentration on Plant Growth: A Mathematical Model, American Journal of Applied Mathematics and Statistics, 2015, Vol. 3, No. 2, 59-67.

[24] Sisay Nune Hailemariam, Teshome Soromessa and Demel Teketay, Non-carbon benefits for effective implementation of REDD+: The case of Bale Mountains Eco-Region, Southeastern Ethiopia, 2015.

[25] Shukla J. B., Freedman, H. I., Pal, V. N., Misra, O. P., Agarwal, M. and Shukla, A., Degradation and subsequent regeneration of a forestry resource: a mathematical model, Ecol. Model., 44, 219-229, 1989.
[26] TerefeDegeti, Factors Affecting PeopleâA Zs Participation in Participatory Forest 'Management: The case of IFMP AdabaDodola in Bale zone of Oromia Region, 2003.

[27] ViviRamdhani, Jaharuddin and E. H. Nugrahani, Dynamical System of Modelling theDepletion of Forestry Resources Due to Crowding by Industrialization, Applied Mathematical Sciences, Vol. 9, 2015, no. 82, 4067-4079.

[28] Waleed K. Ahmed, Advantages and Disadvantages of Using MATLAB/ode45 for Solving Differential (3) Equations in Engineering Applications International Journal of Engineering (IJE), Volume (7): Issue (1): 2013.

[29] KusumLata, Arvind Kumar Misra, Modeling the effect of economic efforts to control population pressure and conserve forestry resources June 19, 2017, Vol. 22, No. 4, 473-488 ISSN 1392-5113.

[30] KusumLata, Arvind Kumar Misra, and Jang Bahadur Shukla. Modeling the effect of deforestation caused by human population pressure on wildlife species. 2018, Vol. 23, No. 3, 303-320 ISSN 1392-5113. 